\title{
Importance of Electronic Correlations and Unusual Excitonic Effects in Formamidinium Lead Halide Perovskites
}

\author{
T. J. Whitcher, ${ }^{1,2, *}$ J.-X. Zhu, ${ }^{3}$ X. Chi, ${ }^{1,4}$ H. Hu,${ }^{5}$ Daming Zhao, ${ }^{6}$ T. C. Asmara, ${ }^{1,7}$ X. Yu, ${ }^{1}$ M. B. H. Breese, ${ }^{1,4}$ \\ A. H. Castro Neto, ${ }^{2}$ Y. M. Lam, ${ }^{5}$ A. T. S. Wee, ${ }^{2,4}$ Elbert E. M. Chia, ${ }^{6, \dagger}$ and A. Rusydi ${ }^{1,4,7,8, \sharp}$ \\ ${ }^{1}$ Singapore Synchrotron Light Source, National University of Singapore, \\ 5 Research Link, Singapore 117603, Singapore \\ ${ }^{2}$ Centre for Advanced 2D Materials, National University of Singapore, Singapore 117546, Singapore \\ ${ }^{3}$ Theoretical Division and Center for Integrated Nanotechnologies, Los Alamos National Laboratory, \\ Los Alamos, New Mexico 87545, USA \\ ${ }^{4}$ Department of Physics, National University of Singapore, Singapore 117542, Singapore \\ ${ }^{5}$ School of Materials Science and Engineering, Nanyang Technological University, \\ 50 Nanyang Avenue, Singapore \\ ${ }^{6}$ Division of Physics and Applied Physics, School of Physical and Mathematical Sciences, \\ Nanyang Technological University, 637371 Singapore \\ ${ }^{7}$ NUSSNI-NanoCore, National University of Singapore, Singapore 117576, Singapore \\ ${ }^{8}$ NUS Graduate School for Integrative Sciences and Engineering, 117456, Singapore
}

(Received 4 October 2017; revised manuscript received 22 January 2018; published 8 May 2018)

\begin{abstract}
Hybrid inorganic-organic perovskites have recently attracted much interest because of both rich fundamental sciences and potential applications such as the primary energy-harvesting material in solar cells. However, an understanding of electronic and optical properties, particularly the complex dielectric function, of these materials is still lacking. Here, we report on the electronic and optical properties of selective perovskites using temperature-dependent spectroscopic ellipsometry, $\mathrm{x}$-ray absorption spectroscopy supported by first-principles calculations. Surprisingly, the perovskite $\mathrm{FA}_{0.85} \mathrm{Cs}_{0.15} \mathrm{PbI}_{2.9} \mathrm{Br}_{0.1}$ has a very high density of low-energy excitons that increases with increasing temperature even at room temperature, which is not seen in any other material. This is found to be due to the strong, unscreened electron-electron and partially screened electron-hole interactions, which then tightly connect low- and high-energy bands caused by doping.
\end{abstract}

DOI: 10.1103/PhysRevX.8.021034

Subject Areas: Materials Science, Optoelectronics, Strongly Correlated Materials

\section{INTRODUCTION}

In recent years, perovskites have been identified as good candidate materials for inexpensive, high-efficiency photovoltaic devices [1-11]. Efficiencies of up to $22 \%$ have already been reported for such devices, while a theoretical limit of $31 \%$ has been postulated $[1,12]$. The most common type of perovskite for these devices is the hybrid inorganicorganic methyl-ammonium lead halide $\left(\mathrm{MAPbX}_{3}\right)$, which has the chemical structure $\mathrm{CH}_{3} \mathrm{NH}_{3} \mathrm{PbX}_{3}$, where $\mathrm{X}=\mathrm{I}, \mathrm{Br}$, or $\mathrm{Cl}$, the most studied of which is $\mathrm{MAPbI}_{3}[5,6]$. It has been

\footnotetext{
*2dwtj@nus.edu.sg

†'ElbertChia@ntu.edu.sg

"phyandri@nus.edu.sg
}

Published by the American Physical Society under the terms of the Creative Commons Attribution 4.0 International license. Further distribution of this work must maintain attribution to the author(s) and the published article's title, journal citation, and DOI. shown through time-resolved terahertz spectroscopy that, at low temperatures, the free-carrier density and mobility of $\mathrm{MAPbI}_{3}$ make it a good candidate for a charge-separation layer in photovoltaic cells [13]. $\mathrm{MAPbI}_{3}$ was found to be unstable at temperatures above $55^{\circ} \mathrm{C}$, partly because of a structural phase transition from the room-temperature tetragonal to the high-temperature cubic phase $[10,11]$, and it is also very susceptible to damage by air and light, forming the yellow $\mathrm{PbI}_{2}[2,3,14]$.

Other perovskites have therefore been developed for efficient solar cells wherein the MA cations have been replaced. These include the inorganic cesium lead halides $\left(\mathrm{CsPbX}_{3}\right)$ [15,16] and formamidinium lead halides, $\mathrm{HC}\left(\mathrm{NH}_{2}\right)_{2} \mathrm{PbX}_{3}\left(\mathrm{FAPbX}_{3}\right)$ [7-9]. Another approach has been to replace the $\mathrm{Pb}$ with other elements such as $\mathrm{Ge}, \mathrm{Si}$, and $\mathrm{Sn}$, with mixed results [17]. All of these changes may affect the physical and electronic structures. For example, by increasing the size of the cation in the perovskite from Cs to MA to FA, the band gap has been decreased from $1.73 \mathrm{eV}$ to $1.57 \mathrm{eV}$ and $1.48 \mathrm{eV}$, respectively [18]. 
However, even $\mathrm{FAPbI}_{3}$ degraded rapidly at room temperature because of the humidity within the air [10], although $\mathrm{CsPbBr} 3$ was relatively stable.

A successful approach has been to mix the perovskite halides and cations in order to achieve stability at higher temperatures and tunability of the band gap $[10,11,19]$. Band-gap tunability from $1.48 \mathrm{eV}$ to $2.23 \mathrm{eV}$ has been achieved via the mixing of bromine and iodine halides [18], while the mixing of $\mathrm{Cs}$ in both $\mathrm{MAPbX}_{3}$ and $\mathrm{FAPbX}_{3}$ perovskites has been shown to improve stability $[10,20]$. That stability was determined by whether the perovskite was in single-crystal bulk form or thin-film nanocrystal or amorphous form. The latter is what is used in solar cells, and it provides a much more realistic interpretation of the physics within the solar cell. However, nanocrystalline thin films degrade much faster, which is why single-crystal perovskites can also be used to determine electrical and optical properties.

Although perovskite solar cells are seen as a significant improvement on conventional solar cells [11], the origin of the density of excitons in the materials is still not well understood. The exciton, a neutral bound electron-hole pair, is a fundamental optical excitation, which is important for both fundamental science and applications such as solar cells [21-23]. Therefore, there is a crucial need to understand the origin of the exciton density and formation in this energyharvesting material. Here, we investigate the electronic and optical properties of thin-film perovskites and find the origin of high excitonic density of $\mathrm{Pb}$-based perovskites. While single-crystal measurements may be useful for the determination of electrical and optical properties at room temperature, at lower temperatures, nanocrystalline effects become important. Hence, in this investigation, we intentionally use thin nanocrystalline films. This is the first temperaturedependent spectroscopic ellipsometry study on $\mathrm{Pb}$-based perovskites, and from these measurements, we are able, for the first time, to determine the cause of the unusual behavior of the excitons at different temperatures.

\section{RESULTS AND DISCUSSION}

The complex dielectric function of multiple samples of three different perovskites was measured using spectroscopic ellipsometry at a wide range of temperatures. Spectroscopic ellipsometry is the most direct way to measure the complex dielectric function, which includes all optically allowed transitions because of the fulfilment of the $f$-sum rule by the measurements due to its full symmetry of polarization in a broad energy range [24-36]. It can simultaneously measure excitons, optical gaps and transitions, and, importantly, spectral-weight transfers [26-28]. By tracking the transfer of spectral weight using spectroscopic ellipsometry, electronelectron correlations, both strong and weak, can be uncovered in a wide range of materials, including correlated electron systems [29-36]. Figures 1(a) and 1(b) show the real and
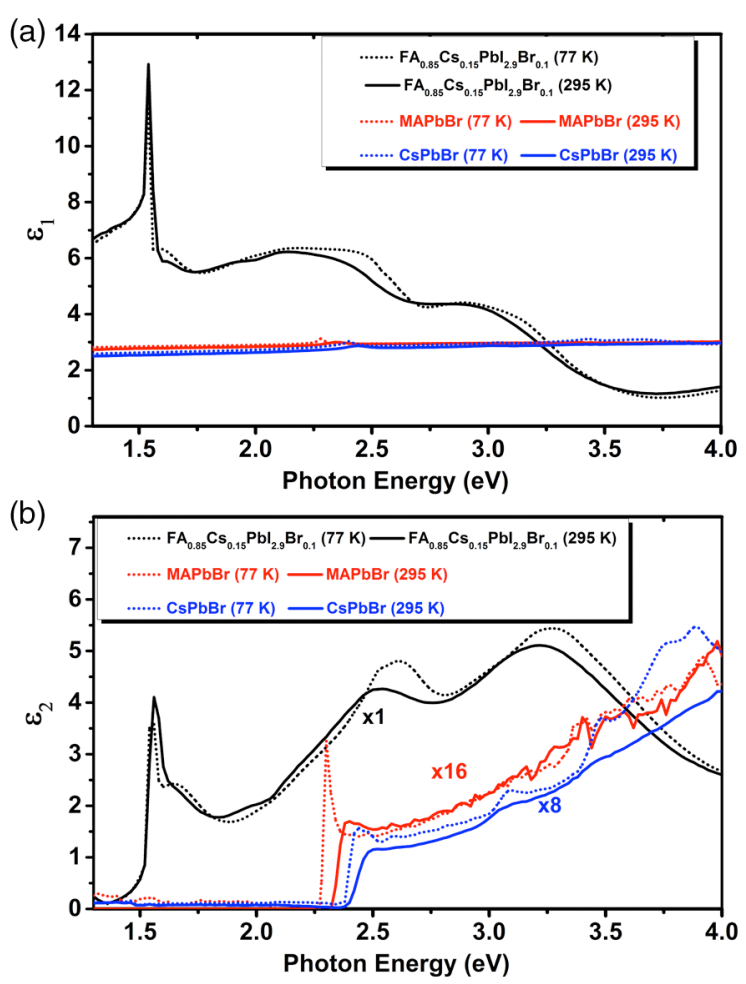

FIG. 1. (a) Comparison of $\varepsilon_{1}$ for $\mathrm{FA}_{0.85} \mathrm{Cs}_{0.15} \mathrm{PbI}_{2.9} \mathrm{Br}_{0.1}$, $\mathrm{MAPbBr}_{3}$, and $\mathrm{CsPbBr}_{3}$ at $77 \mathrm{~K}$ and $295 \mathrm{~K}$. (b) Comparison of the normalized exciton amplitude in $\varepsilon_{2}$ for $\mathrm{FA}_{0.85} \mathrm{Cs}_{0.15}$ $\mathrm{PbI}_{2.9} \mathrm{Br}_{0.1}, \mathrm{MAPbBr}_{3}$, and $\mathrm{CsPbBr}_{3}$ at $77 \mathrm{~K}$ and $295 \mathrm{~K}$. The $\varepsilon_{2}$ data for all of the materials have been normalized, whereby the $\mathrm{CsPbBr}_{3}$ data were increased by a factor of 8 and the $\mathrm{MAPbBr}_{3}$ data were increased by a factor of 16 .

imaginary parts, $\varepsilon_{1}$ and $\varepsilon_{2}$, of the dielectric function of MAPbBr $3, \mathrm{FA}_{0.85} \mathrm{Cs}_{0.15} \mathrm{PbI}_{2.9} \mathrm{Br}_{0.1}$, and $\mathrm{CsPbBr}_{3}$ at room temperature and at $77 \mathrm{~K}$. We chose $\mathrm{FA}_{0.85} \mathrm{Cs}_{0.15} \mathrm{PbI}_{2.9} \mathrm{Br}_{0.1}$ in these experiments, as measuring $\mathrm{FAPbI}_{3}$ is very difficult because of the rapid degradation of the thin film without a capping layer [10]. $\mathrm{FA}_{0.85} \mathrm{Cs}_{0.15} \mathrm{PbI}_{2.9} \mathrm{Br}_{0.1}$ is a stable and viable alternative that has proven to have a high efficiency $[10,11,19]$.

Interestingly, in $\mathrm{FA}_{0.85} \mathrm{Cs}_{0.15} \mathrm{PbI}_{2.9} \mathrm{Br}_{0.1}$, the spectra reveal strong excitonic effects and very high absorption. At low energy, $\varepsilon_{1}$ of $\mathrm{FA}_{0.85} \mathrm{Cs}_{0.15} \mathrm{PbI}_{2.9} \mathrm{Br}_{0.1}$ is very high, almost twice that of $\mathrm{MAPbBr}_{3}$ and $\mathrm{CsPbBr}_{3}$. This reveals that the electron-electron interaction plays an important role in determining the electronic structure and exciton density of $\mathrm{FA}_{0.85} \mathrm{Cs}_{0.15} \mathrm{PbI}_{2.9} \mathrm{Br}_{0.1}$. Although $\varepsilon_{1}$ of $\mathrm{FA}_{0.85}$ $\mathrm{Cs}_{0.15} \mathrm{PbI}_{2.9} \mathrm{Br}_{0.1}$ is much larger than the other two perovskites, there is a clear excitonic peak in each case, which can also be seen in Fig. 1(b). The $\varepsilon_{2}$ data for all of the materials have been normalized with respect to the $\mathrm{FA}_{0.85} \mathrm{Cs}_{0.15} \mathrm{PbI}_{2.9} \mathrm{Br}_{0.1}$ because of the generally high dielectric function of the material. It is clear that $\mathrm{FA}_{0.85} \mathrm{Cs}_{0.15} \mathrm{PbI}_{2.9} \mathrm{Br}_{0.1}$ has the highest intensity excitonic peak. The energy of the exciton of $\mathrm{FA}_{0.85} \mathrm{Cs}_{0.15} \mathrm{PbI}_{2.9} \mathrm{Br}_{0.1}$ 

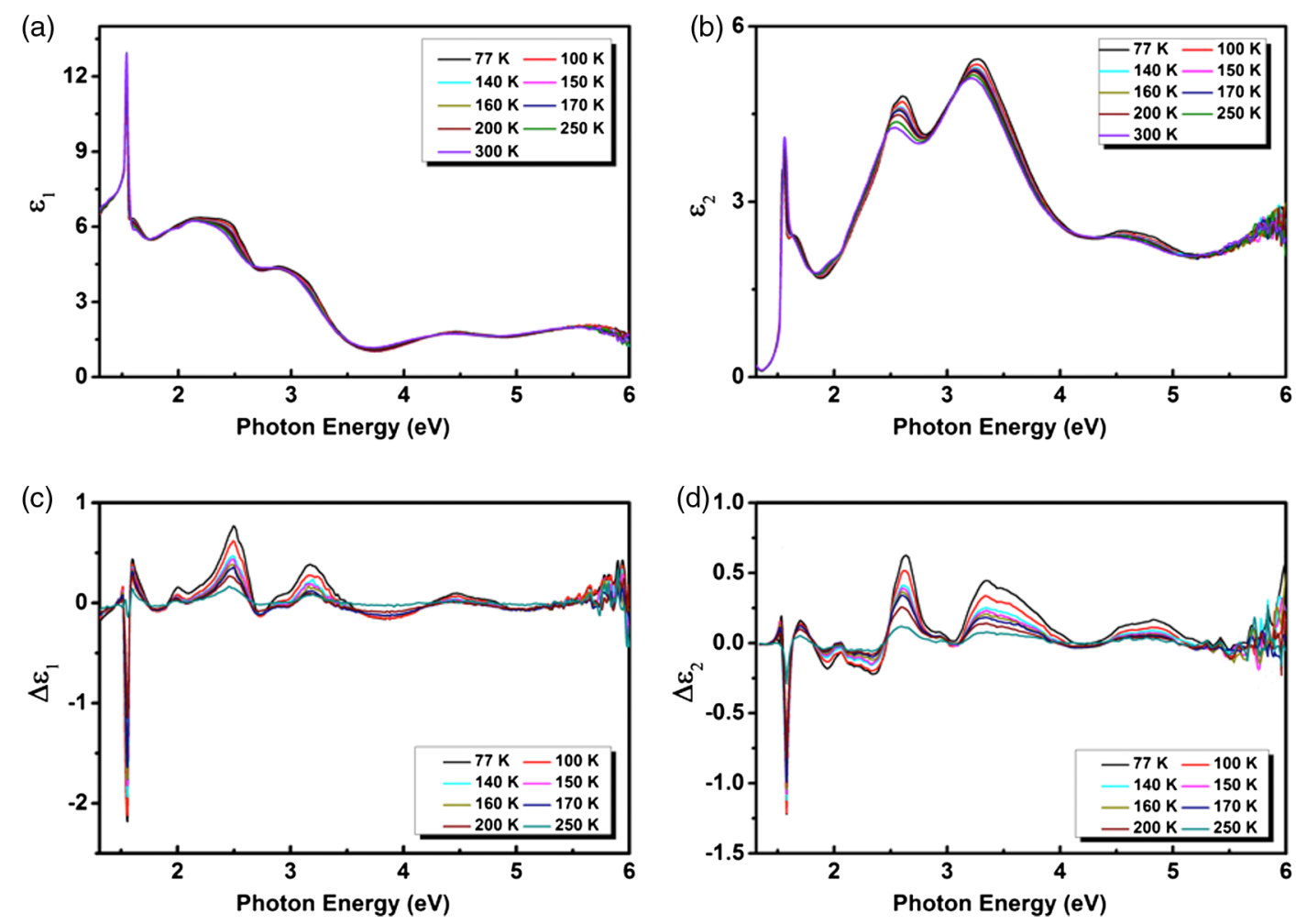

FIG. 2. (a) The real $\left(\varepsilon_{1}\right)$ and (b) imaginary $\left(\varepsilon_{2}\right)$ parts of the dielectric function of $\mathrm{FA}_{0.85} \mathrm{Cs}_{0.15} \mathrm{PbI}_{2.9} \mathrm{Br}_{0.1}$ modeled from spectroscopic ellipsometry data as a function of temperature. The difference in the (c) real and (d) imaginary parts of the dielectric function between room temperature and colder measurements.

is at about $1.55 \mathrm{eV}$, which is higher than the $1.47-\mathrm{eV}$ exciton of $\mathrm{FAPbI}_{3}[7-9,14]$ and is lower than the nearly $2.3-\mathrm{eV}$ exciton of $\mathrm{MAPbBr}_{3}$ and the nearly $2.45-\mathrm{eV}$ exciton of $\mathrm{Cs}_{\mathrm{PbBr}}$. Surprisingly, the $\mathrm{FA}_{0.85} \mathrm{Cs}_{0.15} \mathrm{PbI}_{2.9} \mathrm{Br}_{0.1}$ exciton amplitude is larger at room temperature than at $77 \mathrm{~K}$ [cf. Fig. 1(b)], implying that most excitons occur at room temperature. This is in contrast to what happens in $\mathrm{MAPbI}_{3}$ [24,25], $\mathrm{MAPbBr}_{3}$, and $\mathrm{CsPbBr}_{3}$, where the number of excitons decreases with increasing temperature. Note that the effect of the mixing in $\mathrm{FAPbX}_{3}$ perovskites has been known to drastically reduce the trap density [10], which would in turn affect the exciton density of the material. This also suggests that the exciton density of $\mathrm{FAPbI}_{3}$ would be even higher than that of $\mathrm{FA}_{0.85} \mathrm{Cs}_{0.15} \mathrm{PbI}_{2.9} \mathrm{Br}_{0.1}$. We next focus our discussion on the unusual excitonic behavior in $\mathrm{FA}_{0.85} \mathrm{Cs}_{0.15} \mathrm{PbI}_{2.9} \mathrm{Br}_{0.1}$.

The complex dielectric function of the $\mathrm{FA}_{0.85} \mathrm{Cs}_{0.15}$ $\mathrm{PbI}_{2.9} \mathrm{Br}_{0.1}$ is measured as a function of temperature from $77 \mathrm{~K}$ up to $300 \mathrm{~K}$ in order to quantitatively determine the behavior of the lattice and the excitonic effects with increasing temperature. The real and imaginary parts of the dielectric function, $\varepsilon_{1}$ and $\varepsilon_{2}$, are shown in Figs. 2(a) and 2(b), respectively. The excitonic feature is present at $1.55 \mathrm{eV}$, with further critical points at around $2.55 \mathrm{eV}$ and $3.25 \mathrm{eV}$. Interestingly, these points are also where the greatest changes with temperature occur. Figures 2(c) and 2(d) show the change in $\varepsilon_{1}$ and $\varepsilon_{2}$, respectively, as the $\mathrm{FA}_{0.85} \mathrm{Cs}_{0.15} \mathrm{PbI}_{2.9} \mathrm{Br}_{0.1}$ is cooled from room temperature to $77 \mathrm{~K}$.

With regards to the evolution of the dielectric function with temperature, the changes are very small, and there are no sudden shifts such as those that occur in $\mathrm{MAPbX}_{3}$ when there is a phase change between the low-temperature orthorhombic phase and the room-temperature tetragonal phase [24]. This strongly suggests that there is no phase change in $\mathrm{FA}_{0.85} \mathrm{Cs}_{0.15} \mathrm{PbI}_{2.9} \mathrm{Br}_{0.1}$ below room temperature that usually occurs in most lead-based perovskites [23,25]. There also appears to be a small energy shift in the critical points with temperature, and this will be discussed later.

Using the results of the spectroscopy ellipsometry, the exciton binding energy and the temperature coefficients of the $\mathrm{FA}_{0.85} \mathrm{Cs}_{0.15} \mathrm{PbI}_{2.9} \mathrm{Br}_{0.1}$ can be extracted (see Ref. [37] for details) [25]. Note that this method of using the temperature dependence of the excitonic peak takes into account the thermal broadening of the peak, which leads to overestimates of the binding energy and avoids the use of magnetic fields such as when used in magneto-optic measurements [38]. The exciton binding energy is found to be $(5.9 \pm 2.0) \mathrm{meV}$. This is small when compared with the estimated binding energy of $(55 \pm 20) \mathrm{meV}$ for $\mathrm{MAPbI}_{3}$ obtained using the same method [25]. However, it compares favorably to the values of $(10 \pm 3) \mathrm{meV}$ for pure $\mathrm{FAPbI}_{3}$ in the tetragonal phase [23]. Such a small exciton binding energy would mean that the conduction mechanism 



FIG. 3. (a) The change in integrated intensity of the imaginary part of the dielectric function as a function of temperature for different spectral ranges. Inset: The change in the integrated intensity across the whole energy spectrum as a function of temperature. (b) Surface plot of the optical conductivity of $\mathrm{FA}_{0.85} \mathrm{Cs}_{0.15} \mathrm{PbI}_{2.9} \mathrm{Br}_{0.1}$ as a function of temperature and photon energy.

of the $\mathrm{FA}_{0.85} \mathrm{Cs}_{0.15} \mathrm{PbI}_{2.9} \mathrm{Br}_{0.1}$ is largely dominated by free carriers-conventionally, one might think that the large numbers of excitons (inferred from Fig. 1) could be easily dissociated by just the thermal energy $\left(\sim k_{B} T\right.$, where $k_{B}$ is the Boltzmann constant and $T$ is temperature) of a system at a temperature of about $70 \mathrm{~K}$ (about $6.0 \mathrm{meV}$ ). However, Figs. 1 and 2(b) show that the exciton density increases with increasing temperature. Using x-ray absorption spectroscopy (see Ref. [37]), core-hole excitons are revealed, which have much higher binding energies of the order of $1 \mathrm{eV}$. Such a high binding energy of core-level excitons reveals the importance of strong electron-electron interactions, which create a high density of low-energy excitons even at room temperature. These excitons are much more localized and require far more energy to create, and they have been found dominantly at the cation of FA and $\mathrm{Cs}^{+}$. In order to determine the origin of the high exciton densities, we need to look at how the spectral weight transfers in a broad energy range as a function of temperature.

Figure 3(a) shows the change in the integrated intensity as the temperature increases from $77 \mathrm{~K}$ to $300 \mathrm{~K}$ for a number of different spectral ranges, along with the total integrated intensity over the whole spectral range shown in the inset. Interestingly, over the entire spectral range, there is a general decrease in intensity by $6.34 \%$. This means that there is a shift in spectral weight to energies higher than $6.5 \mathrm{eV}$, beyond our experimental data. The spectral weight transfer in such a broad energy range is a fingerprint of strong electronic correlations and is well known to occur in so-called strongly correlated electron systems [26-33]. Surprisingly, the spectral range that contains the excitonic peak shows an increase in spectral weight due to the increase in intensity of the peak with temperature, as shown in Fig. 2(b). The general spectral weight loss with the increased temperature mainly occurs from $2.50 \mathrm{eV}$ to higher energies, which means that there is an injection of more than $2.50 \mathrm{eV}$ of energy into the system. This cannot be attributed to the thermal fluctuations caused by the increase in temperature from $77 \mathrm{~K}$ to $300 \mathrm{~K}$ as that would only add about $18 \mathrm{meV}$ of kinetic energy to the system. Therefore, the increase in energy must come from potential energy, i.e., Coulomb repulsion and electron-electron correlation.

This is further highlighted by Fig. 3(b), which shows the optical conductivity spectra of the $\mathrm{FA}_{0.85} \mathrm{Cs}_{0.15} \mathrm{PbI}_{2.9} \mathrm{Br}_{0.1}$ as the temperature is changed. The optical conductivity is directly related to the number of electrons in the system and shows a clear shift in the spectral weight of the peaks at about $2.50 \mathrm{eV}$ and $3.30 \mathrm{eV}$ to the low-energy excitonic peak as the temperature is increased. Therefore, the complex dielectric function directly reveals that electronic correlations play an important role in the excitonic features and optical spectra of $\mathrm{FA}_{0.85} \mathrm{Cs}_{0.15} \mathrm{PbI}_{2.9} \mathrm{Br}_{0.1}$. As such, the unusual excitonic effects appear to occur with the introduction of FA in the place of MA or Cs within the perovskite; thus, this is likely the main influencing factor. This also sets $\mathrm{FA}_{0.85} \mathrm{Cs}_{0.15} \mathrm{PbI}_{2.9} \mathrm{Br}_{0.1}$ apart from other excitonic systems such as $\mathrm{ZnO}$ whose excitons are independent of electron-electron correlation.

In order to identify the critical points seen in the complex dielectric function in Fig. 2, we use simulated energy dispersion bands from density functional theory (DFT) band-structure calculations of the material. For illustration, Fig. 4(a) shows the energy dispersion bands of $\mathrm{MAPbI}_{3}$ taken from Ref. [39]. Mixed halide formamidinium lead perovskites have previously been shown to have a tetragonal structure when the halide ratio is in favor of Iodine, while the perovskites exhibit a cubic structure when bromine is more heavily featured $[11,18]$. Therefore, this $\mathrm{MAPbI}_{3}$ band structure is used, as it is based on a bodycentered tetragonal Brillouin zone [shown in Fig. 4(b)], whereas previous simulations of $\mathrm{FAPbI}_{3}$ are calculated using a simple cubic Brillouin zone [40]. This can even be used for the lower temperatures due to the lack of phase change seen in the dielectric function. Other factors that also support using this band structure are the similarities in the size of the energy band gaps of the $\mathrm{FA}_{0.85} \mathrm{Cs}_{0.15} \mathrm{PbI}_{2.9}$ $\mathrm{Br}_{0.1}$ and $\mathrm{MAPbI}_{3}$.

The gamma point seen in Fig. 4(a) is measured to be about $1.55 \mathrm{eV}$, which is in the proximity of the exciton 
(a)

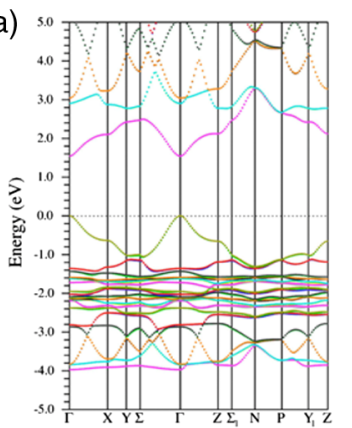

(b)



(c)

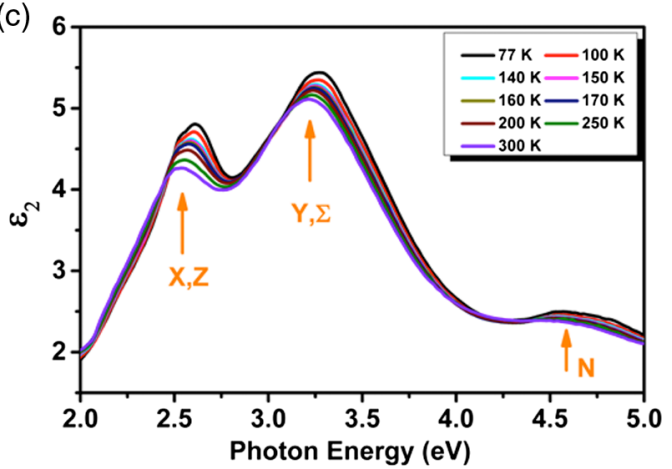

FIG. 4. (a) Energy dispersion bands of $\mathrm{MAPbI}_{3}$ from DFT simulations from Ref. [39]. (b) Body-centered tetragonal Brillouin zone. (c) Imaginary part of the dielectric function showing the higher-energy critical points matched to the energy transitions from simulations.

energy we have measured from spectroscopic ellipsometry. This also shows that the excitonic binding energy is weak, as revealed earlier. The first critical point shown in Fig. 4(c), which has an energy of 2.5-2.6 eV, coincides closely with the energy gaps at both the $X$ and $Z$ points of the Brillouin zone. If the $\Gamma$ point is where the $\mathrm{Pb}$ ion is located within the perovskites, then the halides ( $\mathrm{I}$ or $\mathrm{Br}$ ) would be located at the $X$ and $Z$ points. This would also fit in with the $\mathrm{MAPbI}_{3}$ simulations in Ref. [39], which show that the contributions to the valence band come from the Pb6s orbital and the I5p $(\mathrm{Br} 4 \mathrm{p})$ orbital, with the former having a more dominant role. The only contribution to the conduction band comes from the Pb6p orbital, so the two transitions would come from the Pb6s to the Pb6p at the $\Gamma$ point, and I5p (Br4p) to the Pb6p at the $X$ and $Z$ points [39]. Although the Pb6s to $\mathrm{Pb} 6 \mathrm{p}$ optical transition is the closest to the excitonic transition, which is consistent with the presence of excitons in most, if not all, $\mathrm{Pb}$-based perovskites, it must be emphasized that the other elements, FA, Cs, I, and Br, all affect the excitonic binding energy and density because the bands of these elements are hybridized with each other and the change in spectral weight reveals that all bands as high as $6.0 \mathrm{eV}$ contribute to the formation of these excitons.

The second critical point at around $3.25 \mathrm{eV}$ matches up to the energy gap at the $Y, Y_{1}$, and $\Sigma$ points, which are all between $3.2 \mathrm{eV}$ and $3.3 \mathrm{eV}$. It is very interesting to note that

we have used the dispersion relation of $\mathrm{MAPbI}_{3}$ to accurately map the energy transitions of the $\mathrm{FA}_{0.85} \mathrm{Cs}_{0.15}$ $\mathrm{PbI}_{2.9} \mathrm{Br}_{0.1}$, showing that this can be a good substitute for $\mathrm{MAPbI}_{3}$ in solar cells because of the similar electronic structure but significantly increased excitonic activity. Although we find that the ground-state electronic structures of both $\mathrm{MAPbI}_{3}$ and $\mathrm{FA}_{0.85} \mathrm{Cs}_{0.15} \mathrm{PbI}_{2.9} \mathrm{Br}_{0.1}$ systems are quite similar and the band-structure calculations may help to identify optical transitions in general, the ground-state calculations do not contain the most important information, i.e., excitons and electronic correlations. Therefore, we must turn to complex dielectric function calculations.

Electronic structure and optical absorption calculations are carried out using density functional theory. The complex dielectric functions of both $\mathrm{FAPbI}_{3}$ and $\mathrm{FA}_{0.85} \mathrm{Cs}_{0.15}$ $\mathrm{PbI}_{2.9} \mathrm{Br}_{0.1}$ are calculated and are then compared with the measured dielectric function of $\mathrm{FA}_{0.85} \mathrm{Cs}_{0.15} \mathrm{PbI}_{2.9} \mathrm{Br}_{0.1}$. Figure 5 shows the comparison of $\varepsilon_{2}$ of the $\mathrm{FA}_{0.85} \mathrm{Cs}_{0.15}$ $\mathrm{PbI}_{2.9} \mathrm{Br}_{0.1}$ data at $77 \mathrm{~K}$ with calculations of (a) $\mathrm{FAPbI}_{3}$ and (b) $\mathrm{FA}_{0.85} \mathrm{Cs}_{0.15} \mathrm{PbI}_{2.9} \mathrm{Br}_{0.1}$, both with and without the Bethe-Salpeter equation (BSE). These comparisons allow us to reveal electron-hole interactions yielding excitonic effects. From our calculations, we clarify the following: First, we find that calculations based on BSE, in general, closely resemble experimental results, implying the importance of both electron-hole and electron-electron interactions on the electronic structure and optical properties of the system. Second, the low-energy optical transition at about $1.55 \mathrm{eV}$ is due to strong intrinsic excitonic effects. Upon doping, the calculations show that the exciton shifts toward higher photon energy (or lower binding energy), which implies that the electron-hole interaction is partially screened, yielding a lower exciton binding energy.

Our SOC-GW + BSE calculations show that the excitonic peaks are much larger in $\mathrm{FAPbI}_{3}$ than they are in $\mathrm{FA}_{0.85} \mathrm{Cs}_{0.15} \mathrm{PbI}_{2.9} \mathrm{Br}_{0.1}$. This reinforces the previous discussion that the introduction of $\mathrm{Cs}$ and $\mathrm{Br}$ in $\mathrm{FAPbI}_{3}$ drastically reduces the trap density and therefore the exciton density [10]. However, the most important conclusion to draw from Fig. 5 is that the inclusion of BSE, which calculates electron-electron and electron-hole correlations, shifts the spectral weight from higher energies to lower energies in both $\mathrm{FAPbI}_{3}$ and $\mathrm{FA}_{0.85} \mathrm{Cs}_{0.15} \mathrm{PbI}_{2.9} \mathrm{Br}_{0.1}$. This includes the increase of the low-energy excitonic peak, which shows that the presence of electron-electron correlations increases the excitonic density. The role of electronic correlations is further revealed as shown in the temperature-dependent optical conductivity (Fig. 3). When the temperature increases, the spectral weight below $2.4 \mathrm{eV}$ increases, accompanied by a decrease in the spectral weight above $2.4 \mathrm{eV}$. This is direct evidence of the importance of electronic correlations. Note that the thermal energy change as a function of temperature from $77 \mathrm{~K}$ to $300 \mathrm{~K}$ is only about $0.02 \mathrm{eV}$; therefore, thermal fluctuation cannot be responsible for such broad spectral weight transfers of up to $6 \mathrm{eV}$. 

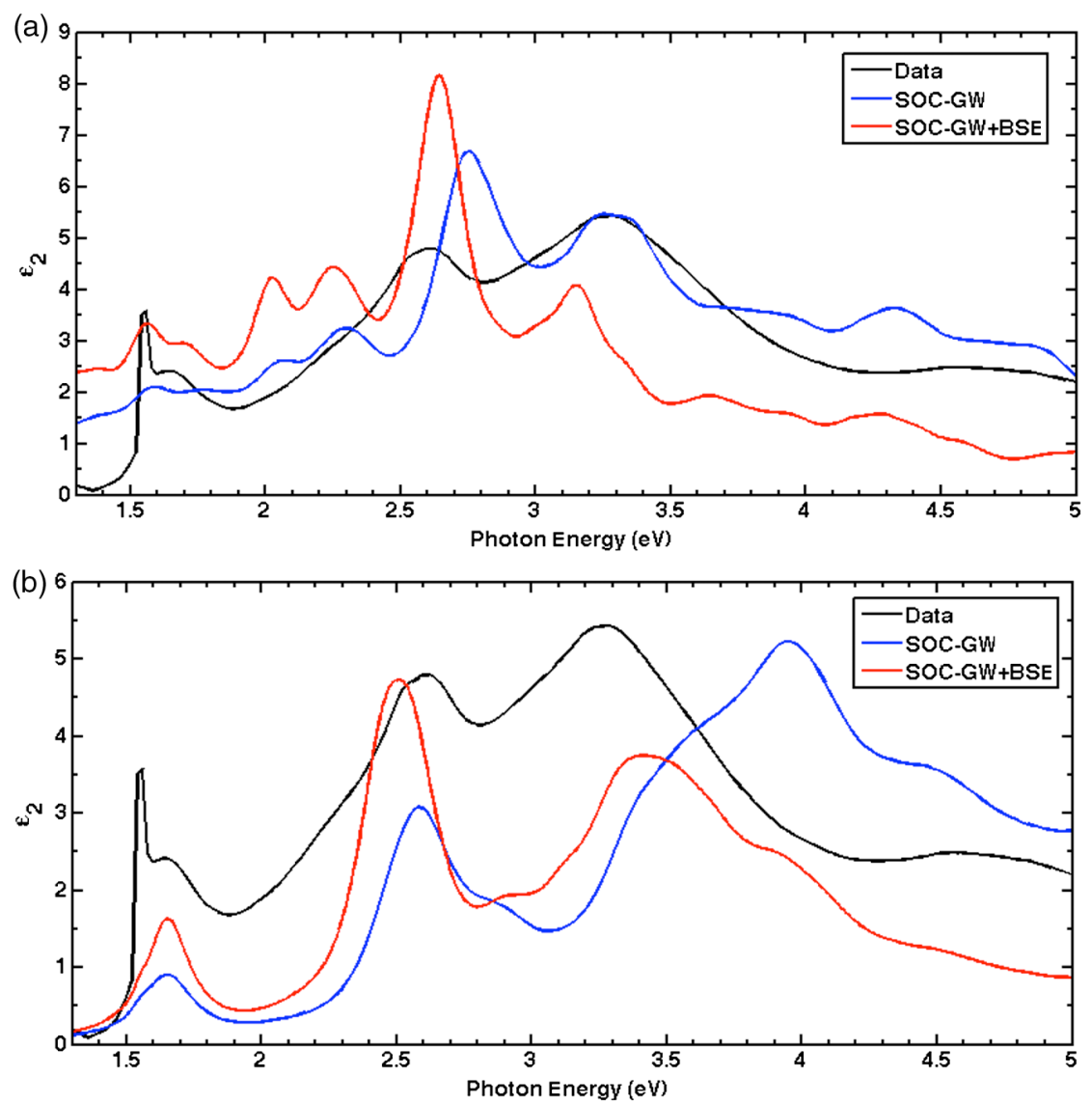

FIG. 5. Comparison of the data at $77 \mathrm{~K}$ with calculations of $\varepsilon_{2}$ from DFT simulations of (a) undoped $\mathrm{FAPbI}_{3}$ and (b) $\mathrm{FA}_{0.85} \mathrm{Cs}_{0.15} \mathrm{PbI}_{2.9} \mathrm{Br}_{0.1}$, both with and without electron-hole interactions (BSE). Note that spin orbit coupling (SOC) is included in our calculations.

The excitonic and optical phenomena in $\mathrm{FA}_{0.85} \mathrm{Cs}_{0.15}$ $\mathrm{PbI}_{2.9} \mathrm{Br}_{0.1}$ are found to be different than that of other systems. In $\mathrm{CsPbBr}_{3}$, the exciton and low-energy excitations have a general reduction in spectral weight with temperature, and in $\mathrm{MAPbBr}_{3}$, they show very little change in spectral weight [cf. Fig. 1(b)]. Generally, an increase in temperature results in the reduction of the excitonic density due to the increase in thermal energy, which allows excitons with low binding energies of less than $50 \mathrm{meV}$ to disassociate more frequently as seen in the $\mathrm{CsPbBr}$ and $\mathrm{MAPbBr}_{3}$. However, it is clear that increasing the temperature in $\mathrm{FA}_{0.85} \mathrm{Cs}_{0.15}$ $\mathrm{PbI}_{2.9} \mathrm{Br}_{0.1}$ increases the electron-electron correlations, which results in a higher exciton density.

Decreasing the temperature in a material usually results in reduced electron screening, which in turn increases electron-electron correlations [26,27]. However, the result of increased electron-electron interactions in $\mathrm{FA}_{0.85} \mathrm{Cs}_{0.15}$ $\mathrm{PbI}_{2.9} \mathrm{Br}_{0.1}$, and $\mathrm{FAPbI}_{3}$, with temperature means that the electronic screening has been reduced. As this effect appears to occur with the introduction of FA in the place of MA or Cs within the perovskite, then this is likely the influencing factor.

It is important to highlight that our spectroscopic ellipsometry data show a sharp, well-defined, low-energy exciton for $\mathrm{FA}_{0.85} \mathrm{Cs}_{0.15} \mathrm{PbI}_{2.9} \mathrm{Br}_{0.1}$, and the presence of the electron-hole interaction is supported by both GW-BSE calculations and XAS. This shows that while the electronhole interaction is partially screened, surprisingly the electron-electron interaction is enhanced. The data above $3.0 \mathrm{eV}$, including the $Y / \Sigma$ peak at $3.3 \mathrm{eV}$, is more closely fit without using BSE, but the more accurate energy fit of the $X / Z$ peak at $2.6 \mathrm{eV}$ using BSE shows that electron-hole interactions have a much bigger effect on this transition. The accurate BSE calculations of both the first interband transition at the $\Gamma$ point and the low-energy exciton peak with experimental data show that the interplay of strong electron-electron and electron-hole interactions determines fundamental properties, yielding high-density excitons in the formamidinium lead halide perovskites.

\section{CONCLUSIONS AND DEVELOPMENTS}

An important fundamental property that is required for the development of perovskite optoelectronics is a high optical absorption in the visible energy range. Here, we reveal that because of strong electronic correlations, the absorption coefficient of $\mathrm{FA}_{0.85} \mathrm{Cs}_{0.15} \mathrm{PbI}_{2.9} \mathrm{Br}_{0.1}$ is much higher than that of $\mathrm{MAPbBr}_{3}$ and $\mathrm{CsPbBr}_{3}$, 



FIG. 6. (a) Comparison of absorption coefficients of $\mathrm{FA}_{0.85} \mathrm{Cs}_{0.15} \mathrm{PbI}_{2.9} \mathrm{Br}_{0.1}, \mathrm{MAPbBr}_{3}$, and $\mathrm{CsPbBr}_{3}$ at different temperatures. (b) Comparison of photoluminescence and the dielectric function $\varepsilon_{2}$ for $\mathrm{FA}_{0.85} \mathrm{Cs}_{0.15} \mathrm{PbI}_{2.9} \mathrm{Br}_{0.1}$ to further support the presence of an exciton at about $1.55 \mathrm{eV}$.

particularly around the excitonic energy of about $1.55 \mathrm{eV}$ as seen in Fig. 6(a). Interestingly, the absorption coefficient of $\mathrm{FA}_{0.85} \mathrm{Cs}_{0.15} \mathrm{PbI}_{2.9} \mathrm{Br}_{0.1}$ is more than 10 times higher than $\mathrm{CH}_{3} \mathrm{NH}_{3} \mathrm{PbI}_{3}$ and $\mathrm{CsSnI}$, as two representatives of halide perovskites, and conventional semiconductors such as GaAs [41]. Such a very high absorption coefficient would allow one to potentially use this material as an effective absorber, even on a very thin film; this is because photons can create a lot of electron-hole pairs (or excitons) at the surface without the need for the photons to travel deeper into the material, which may then also avoid a low nonradiative recombination rate. To further support the presence of excitons in our $\mathrm{FA}_{0.85} \mathrm{Cs}_{0.15} \mathrm{PbI}_{2.9} \mathrm{Br}_{0.1}$ sample, room-temperature photoluminescence measurements shown in Fig. 6(b) clearly exhibit an absorption peak where the excitons occur in $\varepsilon_{2}$. Our results show the importance of electronic correlations in determining excitonic effects, which is crucial in increasing the efficiency of perovskite photovoltaic devices through the manipulation of the excitonic density and will have a profound impact on the way light-harvesting materials are being used in optoelectronic devices.

\section{ACKNOWLEDGMENTS}

This work is supported by the Ministry of Education (Grants No. MOE2015-T2-2-065, No. MOE2015-T2-1099, and No. MOE2015-T2-2-147), Singapore National Research Foundation under its Competitive Research Funding (Grants No. NRF-CRP 8-2011-06, No. R-398000-087-281, and No. NRF-CRP15-2015-01) and under its Medium Sized Centre Programme (Centre for Advanced 2D Materials and Graphene Research Centre), NUS YIA, Faculty Research Committee (FRC) (Grants No. R-144000-379-114 and No. R-144-000-368-112), and NUS Core Support Grant No. C-380-003-003-001. This work was supported, in part, by the Center for Integrated Nanotechnologies, a U.S. DOE Office of Basic Energy Sciences user facility, in partnership with the LANL Institutional Computing Program for computational resources.

T. J. W., J.-X.Z, and X. C. contributed equally to this work.

\section{APPENDIX: EXPERIMENTAL DETAILS}

\section{Sample preparation}

$\operatorname{Poly}\left(N, \quad N^{\prime}\right.$-bis(4-butylphenyl)- $N, \quad N^{\prime}$-bis(phenyl)benzidine) (poly-TPD) and bathocuproine (BCP) were purchased from American Dye Source and Lumtec, respectively. $\mathrm{PbI}_{2}$ and $\mathrm{PbBr}_{2}$ were purchased from TCI (Tokyo Chemical Industry). Other chemicals were purchased from Sigma-Aldrich. PCBM (60) was purchased from Nano-C. Formamidinium iodide (FAI) was synthesized according to the reported method [18]. The crude material was dissolved in ethanol and recrystallized at $-3^{\circ} \mathrm{C}$ overnight. Perovskite precursor solution $\left(\mathrm{FA}_{0.85} \mathrm{Cs}_{0.15} \mathrm{PbI}_{2.9} \mathrm{Br}_{0.1}\right)$ was prepared by dissolving FAI, CsI, $\mathrm{PbI}_{2}$, and $\mathrm{PbBr}_{2}$ with a respective stoichiometric ratio in dimethylformamide/dimethyl sulfoxide $(4: 1 \mathrm{v} / \mathrm{v})$ at $80^{\circ} \mathrm{C}$. The perovskite solution $(1 \mathrm{M})$ was spin coated on z-cut quartz at $1000 \mathrm{rpm}$ for $10 \mathrm{~s}$ and $4000 \mathrm{rpm}$ for $30 \mathrm{~s}$, and $250 \mu \mathrm{L}$ chlorobenzene was dripped on the sample surface at $15 \mathrm{~s}$ of the second-step spin coating. Subsequently, the perovskite layer was annealed at $80^{\circ} \mathrm{C}$ for $5 \mathrm{~min}, 120^{\circ} \mathrm{C}$ for $5 \mathrm{~min}$, and $180^{\circ} \mathrm{C}$ for $30 \mathrm{~min}$. The perovskite layer was measured to be $350 \mathrm{~nm}$ through spectroscopic ellipsometry.

The perovskite solar cell was prepared on precleaned patterned indium tin oxide (ITO) substrate. Poly-TPD was spin coated from solution $(6 \mathrm{mg} / \mathrm{ml}$ in chlorobenzene) at $4000 \mathrm{rpm}$ for $40 \mathrm{~s}$, followed by baking at $120^{\circ} \mathrm{C}$ for $20 \mathrm{~min}$. Prior to the perovskite coating, the poly-TPD surface was treated in UV-ozone cleaner for $10 \mathrm{~s}$. The perovskite precursor solution was spin coated onto poly-TPD at $1000 \mathrm{rpm}$ for $10 \mathrm{~s}$ and $4000 \mathrm{rpm}$ for $30 \mathrm{~s}$, and $250 \mu \mathrm{L}$ chlorobenzene was dripped on the sample surface at $15 \mathrm{~s}$ of the second-step spin coating. Subsequently, the perovskite layer was annealed at $80^{\circ} \mathrm{C}$ for $5 \mathrm{~min}, 120^{\circ} \mathrm{C}$ for $5 \mathrm{~min}$, and 
$180^{\circ} \mathrm{C}$ for $30 \mathrm{~min}$. The PCBM solution was coated from chlorobenzene solution $(20 \mathrm{mg} / \mathrm{mL})$ at $1000 \mathrm{rpm}$ for $60 \mathrm{~s}$. The devices were completed by evaporating BCP $(6 \mathrm{~nm})$ and $\mathrm{Ag}(120 \mathrm{~nm})$ sequentially under high vacuum $\left(1 \times 10^{-6}\right.$ mbar $)$. The active area was $7 \mathrm{~mm}^{2}$ as defined by the overlapping between the back electrode and ITO.

\section{Spectroscopic ellipsometry}

Spectroscopic ellipsometry measurements were carried out using a variable-angle spectroscopic ellipsometer (V-VASE, J. A. Woollam Co.) with a rotating analyzer and compensator on multiple samples. The measurements were taken in the energy range of $0.6-6.0 \mathrm{eV}$, while the samples were inside an ultrahigh vacuum cryostat with a base pressure of $10^{-8} \mathrm{mbar}$. The samples were cooled to $77 \mathrm{~K}$ using liquid nitrogen, and measurements were taken at angles of $68^{\circ}, 70^{\circ}$, and $72^{\circ}$, which are limited in range by the UHV windows. The samples were then heated to temperatures of $100 \mathrm{~K}, 140 \mathrm{~K}, 150 \mathrm{~K}, 160 \mathrm{~K}, 170 \mathrm{~K}, 200 \mathrm{~K}$, $250 \mathrm{~K}$, and room temperature, where measurements were taken at a single angle of $70^{\circ}$ for each temperature. The analysis of the ellipsometry data was performed using the $\mathrm{W}$-VASE analysis program, where the complex dielectric function was obtained using a fitting procedure that models the data.

\section{X-ray absorption spectroscopy}

$\mathrm{X}$-ray absorption spectroscopy (XAS) was performed on the Surface, Interface and Nano-Structure (SINS) beam line at the Singapore Synchrotron Light Source (SSLS). The measurements were carried out ex situ in a UHV chamber with a base pressure of $10^{-10} \mathrm{mbar}$ at room temperature. The beam line uses a modified dragon-type monochromator with spherical gratings that directs the $\mathrm{x}$ rays onto the sample at an angle of $90^{\circ}$. The XAS spectra were measured in total electron yield mode (TEY) by recording the sample drain current. Because of the low effective escape depth of electrons from semiconductors and insulators, this mode of absorption spectroscopy is ideal for surface and interface analysis. In order to correct for intensity fluctuations from the synchrotron, the spectra were normalized to the photoelectron current on the refocusing mirror with gold coating upstream of the samples. The data for spectral ranges that covered the $\mathrm{C} 1 \mathrm{~s}, \mathrm{~N} 1 \mathrm{~s}$, and $\mathrm{Cs} 3 \mathrm{~d}$ lines were recorded at a resolution of $0.2 \mathrm{eV}$.

\section{Electronic structure calculations}

All electronic structure calculations were carried out within the density functional theory (DFT) as implemented in the Vienna $A b$ initio Simulation Package (VASP) [42]. More details of the calculations are given in Ref. [37]. We started the calculations with the atomic position optimization on a cubic $\mathrm{FAPbI}_{3}$ structure with fixed lattice parameters $a=b=c=6.33 \AA$, consistent with the averaged lattice parameter obtained by the DFT calculations reported in previous work using the ultrasoft pseudopotentials and comparable with the experiment result for alpha-FAPbI ${ }_{3}$ [43]. We constructed a supercell of size $2 a \times$ $2 b \times 2 c$, which amounts to eight chemical formula units of $\mathrm{FAPbI}_{3}$, and we used a $2 \times 2 \times 2$ Monkhorst-Pack grid (together with a 500-eV cutoff energy for the plane-wave basis set) to perform the SR-DFT-based optimization of atomic positions for both $\mathrm{FAPbI}_{3}$ and $\mathrm{FA}_{0.875} \mathrm{Cs}_{0.125}$ $\mathrm{PbI}_{2.875} \mathrm{Br}_{0.125}$. For the optical property calculations based on the SOC-GW and SOC-GW + BSE schemes, we limited ourselves to a $2 \times 2 \times 2 \Gamma$-centered $\mathbf{k}$ grid (together with a $200-\mathrm{eV}$ plane-wave cutoff) because of the very expensive computational requirement.

\section{Photoluminescence spectroscopy}

The photoluminescence spectrum was measured by a Cary Eclipse fluorescence spectrophotometer, inside of which is a flashlight containing a broad wavelength. The excitation beam goes through a monochromator. For this measurement, the excitation wavelength is around $550 \mathrm{~nm}$, with a 5-nm slit.

[1] A. Kojima, K. Teshima, Y. Shirai, and T. Miyasaka, Organometal Halide Perovskites as Visible Light Sensitizers for Photovoltaic Cells, J. Am. Chem. Soc. 131, 6050 (2009).

[2] H. S. Kim, C. R. Lee, J. H. Im, K. B. Lee, T. Moehl, A. Marchioro, S. J. Moon, R. Humphry-Baker, J. H. Yum, J. E. Moser, and M. Grätzel, Lead Iodide Perovskite Sensitized All-Solid-State Submicron Thin Film Mesoscopic Solar Cell with Efficiency Exceeding 9\%, Sci. Rep. 2, 591 (2012).

[3] M. M. Lee, J. Teuscher, T. Miyasaka, T. N. Murakami, and H. J. Snaith, Efficient Hybrid Solar Cells Based on MesoSuperstructured Organometal Halide Perovskites, Science 338, 643 (2012).

[4] J. H. Heo, S. H. Im, J. H. Noh, T. N. Mandal, C. S. Lim, J. A. Chang, Y. H. Lee, H. J. Kim, A. Sarkar, M. K. Nazeeruddin, and M. Grätzel, Efficient Inorganic-Organic Hybrid Heterojunction Solar Cells Containing Perovskite Compound and Polymeric Hole Conductors, Nat. Photonics 7, 486 (2013).

[5] J. Burschka, N. Pellet, S. J. Moon, R. Humphry-Baker, P. Gao, M. K. Nazeeruddin, and M. Grätzel, Sequential Deposition as a Route to High-Performance PerovskiteSensitized Solar Cells, Nature (London) 499, 316 (2013).

[6] M. Liu, M. B. Johnston, and H. J. Snaith, Efficient Planar Heterojunction Perovskite Solar Cells by Vapour Deposition, Nature (London) 501, 395 (2013).

[7] S. Aharon, A. Dymshits, A. Rotem, and L. Etgar, Temperature Dependence of Hole Conductor Free Formamidinium Lead Iodide Perovskite Based Solar Cells, J. Mater. Chem. 3, 9171 (2015).

[8] S. Pang, H. Hu, J. Zhang, S. Lu, Y. Yu, F. Wei, T. Qin, H. $\mathrm{Xu}, \mathrm{Z}$. Liu, and G. Cui, $\mathrm{NH}_{2} \mathrm{CHNH}_{2} \mathrm{PbI}_{3}$ : An Alternative 
Organolead Iodide Perovskite Sensitizer for Mesoscopic Solar Cells, Chem. Mater. 26, 1485 (2014).

[9] T. M. Koh, K. Fu, Y. Fang, S. Chen, T. C. Sum, N. Mathews, S. G. Mhaisalkar, P. P. Boix, and T. Baikie, FormamidiniumContaining Metal-Halide: An Alternative Material for Near-IR Absorption Perovskite Solar Cells, J. Phys. Chem. C 118, 16458 (2014).

[10] J. W. Lee, D. H. Kim, H. S. Kim, S. W. Seo, S. M. Cho, and N. G. Park, Formamidinium andcesium Hybridization for Photo-and Moisture-Stable Perovskite Solar Cell, Adv. Energy Mater. 5 (2015).

[11] D. P. McMeekin, G. Sadoughi, W. Rehman, G. E. Eperon, M. Saliba, M. T. Hörantner, A. Haghighirad, N. Sakai, L. Korte, B. Rech, and M. B. Johnston, A Mixed-Cation Lead Mixed-Halide Perovskite Absorber for Tandem Solar Cells, Science 351, 151 (2016).

[12] S. Y. Leblebici, L. Leppert, Y. Li, S. E. Reyes-Lillo, S. Wickenburg, E. Wong, J. Lee, M. Melli, D. Ziegler, D. K. Angell, and D. F. Ogletree, Facet-Dependent Photovoltaic Efficiency Variations in Single Grains of Hybrid Halide Perovskite, Nat. Energy 1, 16093 (2016).

[13] C. La-o-vorakiat, T. Salim, J. Kadro, M. T. Khuc, R. Haselsberger, L. Cheng, H. Xia, G. G. Gurzadyan, H. Su, Y. M. Lam, and R.A. Marcus, Elucidating the Role of Disorder and Free-Carrier Recombination Kinetics in $\mathrm{CH}_{3} \mathrm{NH}_{3} \mathrm{PbI}_{3}$ Perovskite Films, Nat. Commun. 6, 7903 (2015).

[14] S. De Wolf, J. Holovsky, S. J. Moon, P. Löper, B. Niesen, M. Ledinsky, F. J. Haug, J. H. Yum, and C. Ballif, Organometallic Halide Perovskites: Sharp Optical Absorption Edge and Its Relation to Photovoltaic Performance, J. Phys. Chem. Lett. 5, 1035 (2014).

[15] R. E. Beal, D. J. Slotcavage, T. Leijtens, A. R. Bowring, R. A. Belisle, W. H. Nguyen, G. F. Burkhard, E. T. Hoke, and M. D. McGehee, Cesium Lead Halide Perovskites with Improved Stability for Tandem Solar Cells, J. Phys. Chem. Lett. 7, 746 (2016).

[16] M. Kulbak, D. Cahen, and G. Hodes, How Important Is the Organic Part of Lead Halide Perovskite Photovoltaic Cells? Efficient $\mathrm{CsPbBr}_{3}$ Cells, J. Phys. Chem. Lett. 6, 2452 (2015).

[17] L. Y. Huang and W. R. Lambrecht, Electronic Band Structure Trends of Perovskite Halides: Beyond $\mathrm{Pb}$ and $\mathrm{Sn}$ to Ge and Si, Phys. Rev. B 93, 195211 (2016).

[18] G. E. Eperon, S. D. Stranks, C. Menelaou, M. B. Johnston, L. M. Herz, and H. J. Snaith, Formamidinium Lead Trihalide: A Broadly Tunable Perovskite for Efficient Planar Heterojunction Solar Cells, Energy Environ. Sci. 7, 982 (2014).

[19] C. Yi, J. Luo, S. Meloni, A. Boziki, N. Ashari-Astani, C. Grätzel, S. M. Zakeeruddin, U. Röthlisberger, and M. Grätzel, Entropic Stabilization of Mixed A-cation ABX 3 Metal Halide Perovskites for High Performance Perovskite Solar Cells, Energy Environ. Sci. 9, 656 (2016).

[20] M. Kulbak, S. Gupta, N. Kedem, I. Levine, T. Bendikov, G. Hodes, and D. Cahen, Cesium Enhances Long-Term Stability of Lead Bromide Perovskite-Based Solar Cells, J. Phys. Chem. Lett. 7, 167 (2016).
[21] N. Onoda-Yamamuro, T. Matsuo, and H. Suga, Dielectric Study of $\mathrm{CH}_{3} \mathrm{NH}_{3} \mathrm{PbX}_{3}(\mathrm{X}=\mathrm{Cl}, \mathrm{Br}, \mathrm{I})$, J. Phys. Chem. Solids 53, 935 (1992).

[22] A. M. Soufiani, F. Huang, P. Reece, R. Sheng, A. Ho-Baillie, and M. A. Green, Polaronic Exciton Binding Energy in Iodide and Bromide Organic-Inorganic Lead Halide Perovskites, Appl. Phys. Lett. 107, 231902 (2015).

[23] K. Galkowski, A. Mitioglu, A. Miyata, P. Plochocka, O. Portugall, G. E. Eperon, J. T. W. Wang, T. Stergiopoulos, S. D. Stranks, H. J. Snaith, and R. J. Nicholas, Determination of the Exciton Binding Energy and Effective Masses for Methylammonium and Formamidinium Lead Tri-halide Perovskite Semiconductors, Energy Environ. Sci. 9, 962 (2016).

[24] P. Löper, M. Stuckelberger, B. Niesen, J. Werner, M. Filipic, S. J. Moon, J. H. Yum, M. Topič, S. De Wolf, and C. Ballif, Complex Refractive Index Spectra of $\mathrm{CH}_{3} \mathrm{NH}_{3} \mathrm{PbI}_{3}$ Perovskite Thin Films Determined by Spectroscopic Ellipsometry and Spectrophotometry, J. Phys. Chem. Lett. 6, 66 (2015).

[25] V. D’Innocenzo, G. Grancini, M. J. Alcocer, A. R. S. Kandada, S. D. Stranks, M. M. Lee, G. Lanzani, H. J. Snaith, and A. Petrozza, Excitons Versus Free Charges in Organo-Lead Tri-halide Perovskites, Nat. Commun. 5, 3586 (2014).

[26] A. Rusydi, R. Rauer, G. Neuber, M. Bastjan, I. Mahns, S. Müller, P. Saichu, B. Schulz, S. G. Singer, A. I. Lichtenstein, and D. Qi, Metal-Insulator Transition in Manganites: Changes in Optical Conductivity up to $22 \mathrm{eV}$, Phys. Rev. B 78, 125110 (2008).

[27] H. Fujiwara, Spectroscopic Ellipsometry: Principles and Applications (John Wiley \& Sons, New York, 2007).

[28] Z. Yong, P. E. Trevisanutto, L. Chiodo, I. Santoso, A. R. Barman, T. C. Asmara, S. Dhar, A. Kotlov, A. Terentjevs, F. D. Sala, and V. Olevano, Emerging Giant Resonant Exciton Induced by Ta Substitution in Anatase TiO 2: A Tunable Correlation Effect, Phys. Rev. B 93, 205118 (2016).

[29] H. Eskes, M. B. J. Meinders, and G. A. Sawatzky, Anomalous Transfer of Spectral Weight in Doped Strongly Correlated Systems, Phys. Rev. Lett. 67, 1035 (1991).

[30] Y. Ohta, K. Tsutsui, W. Koshibae, T. Shimozato, and S. Maekawa, Evolution of the In-Gap State in High- $\mathrm{T}_{c}$ Cuprates, Phys. Rev. B 46, 14022 (1992).

[31] M. B. J. Meinders, H. Eskes, and G. A. Sawatzky, Spectral-Weight Transfer: Breakdown of Low-Energy-Scale Sum Rules in Correlated Systems, Phys. Rev. B 48, 3916 (1993).

[32] P. Phillips, Colloquium: Identifying the Propagating Charge Modes in Doped Mott Insulators, Rev. Mod. Phys. 82, 1719 (2010).

[33] A. Rusydi, A. Goos, S. Binder, A. Eich, K. Botril, P. Abbamonte, X. Yu, M. B. H. Breese, H. Eisaki, Y. Fujimaki, and S. Uchida, Electronic Screening-Enhanced Hole Pairing in Two-Leg Spin Ladders Studied by High-Resolution Resonant Inelastic X-Ray Scattering at Cu M Edges, Phys. Rev. Lett. 113, 067001 (2014).

[34] D. N. Basov, R. D. Averitt, D. Van Der Marel, M. Dressel, and K. Haule, Electrodynamics of Correlated Electron Materials, Rev. Mod. Phys. 83, 471 (2011). 
[35] A. V. Boris, N. N. Kovaleva, S.S. A. Seo, J. S. Kim, P. Popovich, Y. Matiks, R. K. Kremer, and B. Keimer, Signatures of Electronic Correlations in Optical Properties of $\mathrm{LaFeAsO}_{1-x} \mathrm{~F}_{x}$, Phys. Rev. Lett. 102, 027001 (2009).

[36] M. M. Qazilbash, M. Brehm, B. G. Chae, P. C. Ho, G. O. Andreev, B. J. Kim, S. J. Yun, A. V. Balatsky, M. B. Maple, F. Keilmann, and H. T. Kim, Mott Transition in $\mathrm{VO}_{2}$ Revealed by Infrared Spectroscopy and Nano-imaging, Science 318, 1750 (2007).

[37] See Supplemental Material at http://link.aps.org/supplemental/ 10.1103/PhysRevX.8.021034 for a description of the analysis of the exciton binding energy and the temperature coefficients of the $\mathrm{FA}_{0.85} \mathrm{Cs}_{0.15} \mathrm{PbI}_{2.9} \mathrm{Br}_{0.1}$, x-ray absorption spectroscopy analysis and details of the electronic structure calculations.

[38] A. Miyata, A. Mitioglu, P. Plochocka, O. Portugall, J. T. W. Wang, S. D. Stranks, H. J. Snaith, and R. J. Nicholas, Direct Measurement of the Exciton Binding Energy and Effective Masses for Charge Carriers in
Organic-Inorganic Tri-halide Perovskites, Nat. Phys. 11, 582 (2015).

[39] R. A. Jishi, O. B. Ta, and A. A. Sharif, Modeling of Lead Halide Perovskites for Photovoltaic Applications, J. Phys. Chem. C 118, 28344 (2014).

[40] Y. Y. Pan, Y. H. Su, C. H. Hsu, L. W. Huang, K. P. Dou, and C. C. Kaun, First-Principles Study on Electronic Structures of $\mathrm{FAPbX}_{3}(\mathrm{X}=\mathrm{Cl}, B r, I)$ Hybrid Perovskites, J. Adv. Nanomater. 1, 33 (2016).

[41] W. J. Yin, T. Shi, and Y. Yan, Unique Properties of Halide Perovskites as Possible Origins of the Superior Solar Cell Performance, Adv. Mater. 26, 4653 (2014).

[42] G. Kresse and J. Hafner, Ab Initio Molecular Dynamics for Liquid Metals, Phys. Rev. B 47, 558 (1993).

[43] M. T. Weller, O. J. Weber, J. M. Frost, and A. Walsh, Cubic Perovskite Structure of Black Formamidinium Lead Iodide, $\alpha$ - $\left[\mathrm{HC}\left(\mathrm{NH}_{2}\right)_{2}\right] \mathrm{PbI}_{3}$, at $298 \mathrm{~K}$, J. Phys. Chem. Lett. 6, 3209 (2015). 